\title{
Fixed point theorems on partial randomness*
}

\author{
Kohtaro Tadaki \\ Research and Development Initiative, Chuo University \\ CREST, JST \\ 1-13-27 Kasuga, Bunkyo-ku, Tokyo 112-8551, Japan \\ E-mail: tadaki@kc.chuo-u.ac.jp \\ http://www2.odn.ne.jp/tadaki/
}

\begin{abstract}
In our former work [K. Tadaki, Local Proceedings of CiE 2008, pp. 425-434, 2008], we developed a statistical mechanical interpretation of algorithmic information theory by introducing the notion of thermodynamic quantities at temperature $T$, such as free energy $F(T)$, energy $E(T)$, and statistical mechanical entropy $S(T)$, into the theory. These quantities are real functions of real argument $T>0$. We then discovered that, in the interpretation, the temperature $T$ equals to the partial randomness of the values of all these thermodynamic quantities, where the notion of partial randomness is a stronger representation of the compression rate by program-size complexity. Furthermore, we showed that this situation holds for the temperature itself as a thermodynamic quantity. Namely, the computability of the value of partition function $Z(T)$ gives a sufficient condition for $T \in(0,1)$ to be a fixed point on partial randomness. In this paper, we show that the computability of each of all the thermodynamic quantities above gives the sufficient condition also. Moreover, we show that the computability of $F(T)$ gives completely different fixed points from the computability of $Z(T)$.
\end{abstract}

Key words: algorithmic randomness, fixed point theorem, partial randomness, Chaitin $\Omega$ number, algorithmic information theory, thermodynamic quantities

AMS subject classifications (2000) 68Q30, 26E40, 03D80, 82B30, 82B03

\section{Introduction}

Algorithmic information theory (AIT, for short) is a framework for applying information-theoretic and probabilistic ideas to recursive function theory. One of the primary concepts of AIT is the program-size complexity (or Kolmogorov complexity) $H(s)$ of a finite binary string $s$, which is defined

\footnotetext{
* A preliminary version of this paper appeared in the Proceedings of the Symposium on Logical Foundations of Computer Science 2009 (LFCS'09), S. Artemov and A. Nerode (Eds.), Lecture Notes in Computer Science, SpringerVerlag, Vol.5407, pp.422-440, January 3-6, 2009, Deerfield Beach, Florida, USA.
} 
as the length of the shortest binary program for the universal self-delimiting Turing machine $U$ to output $s$. By the definition, $H(s)$ is thought to represent the degree of randomness of a finite binary string $s$. In particular, the notion of program-size complexity plays a crucial role in characterizing the randomness of an infinite binary string, or equivalently, a real number.

In [19] we developed a statistical mechanical interpretation of AIT. In the development we introduced especially the notion of thermodynamic quantities, such as partition function $Z(T)$, free energy $F(T)$, energy $E(T)$, statistical mechanical entropy $S(T)$, and specific heat $C(T)$, into AIT. These quantities are real numbers which depend on temperature $T$, any positive real number. We then proved that if the temperature $T$ is a computable real number with $0<T<1$ then, for each of these thermodynamic quantities, the partial randomness of its value equals to $T$, where the notion of partial randomness is a stronger representation of the compression rate by means of program-size complexity. Thus, the temperature $T$ plays a role as the partial randomness of all the thermodynamic quantities in the statistical mechanical interpretation of AIT. In [19] we further showed that the temperature $T$ plays a role as the partial randomness of the temperature $T$ itself, which is a thermodynamic quantity of itself. Namely, we proved the fixed point theorem on partial randomness, ${ }^{1}$ which states that, for every $T \in(0,1)$, if the value of partition function $Z(T)$ at temperature $T$ is a computable real number, then the partial randomness of $T$ equals to $T$, and therefore the compression rate of $T$ equals to $T$, i.e., $\lim _{n \rightarrow \infty} H\left(T_{n}\right) / n=T$, where $T_{n}$ is the first $n$ bits of the base-two expansion of $T$.

In this paper, we show that a fixed point theorem of the same form as for $Z(T)$ holds also for each of free energy $F(T)$, energy $E(T)$, and statistical mechanical entropy $S(T)$. Moreover, based on the statistical mechanical relation $F(T)=-T \log _{2} Z(T)$, we show that the computability of $F(T)$ gives completely different fixed points from the computability of $Z(T)$.

The paper is organized as follows. We begin in Section 2 with some preliminaries to AIT and partial randomness. In Section 3, we review the previous results [19] on the statistical mechanical interpretation of AIT and the fixed point theorem by $Z(T)$, which is given as Theorem 3.4 in the present paper. Our main results; the fixed point theorems by $F(T), E(T)$, and $S(T)$, are presented in Section 4, and their proofs are completed in Section 5. In the last section, we investigate some properties of the sufficient conditions for $T$ to be a fixed point in the fixed point theorems.

\section{Preliminaries}

\subsection{Basic notation}

We start with some notation about numbers and strings which will be used in this paper. $\mathbb{N}=$ $\{0,1,2,3, \ldots\}$ is the set of natural numbers, and $\mathbb{N}^{+}$is the set of positive integers. $\mathbb{Q}$ is the set of rational numbers, and $\mathbb{R}$ is the set of real numbers. Let $f: S \rightarrow \mathbb{R}$ with $S \subset \mathbb{R}$. We say that $f$ is increasing (resp., decreasing) if $f(x)<f(y)$ (resp., $f(x)>f(y)$ ) for all $x, y \in S$ with $x<y$. We denote by $f^{\prime}$ the derived function of $f$.

Normally, $o(n)$ denotes any function $f: \mathbb{N}^{+} \rightarrow \mathbb{R}$ such that $\lim _{n \rightarrow \infty} f(n) / n=0$. On the other hand, $O(1)$ denotes any function $g: \mathbb{N}^{+} \rightarrow \mathbb{R}$ such that there is $C \in \mathbb{R}$ with the property that $|g(n)| \leq C$ for all $n \in \mathbb{N}^{+}$.

$\{0,1\}^{*}=\{\lambda, 0,1,00,01,10,11,000, \ldots\}$ is the set of finite binary strings, where $\lambda$ denotes the empty string. For any $s \in\{0,1\}^{*},|s|$ is the length of $s$. A subset $S$ of $\{0,1\}^{*}$ is called prefix-free if

\footnotetext{
${ }^{1}$ The fixed point theorem on partial randomness is called a fixed point theorem on compression rate in [19].
} 
no string in $S$ is a prefix of another string in $S$. For any partial function $f$, the domain of definition of $f$ is denoted by $\operatorname{dom} f$. We write "r.e." instead of "recursively enumerable."

Let $\alpha$ be an arbitrary real number. $\lfloor\alpha\rfloor$ is the greatest integer less than or equal to $\alpha$, and $\lceil\alpha\rceil$ is the smallest integer greater than or equal to $\alpha$. For any $n \in \mathbb{N}^{+}$, we denote by $\alpha_{n} \in\{0,1\}^{*}$ the first $n$ bits of the base-two expansion of $\alpha-\lfloor\alpha\rfloor$ with infinitely many zeros. For example, in the case of $\alpha=5 / 8, \alpha_{6}=101000$.

We say that a real number $\alpha$ is computable if there exists a total recursive function $f: \mathbb{N}^{+} \rightarrow \mathbb{Q}$ such that $|\alpha-f(n)|<1 / n$ for all $n \in \mathbb{N}^{+}$. We say that $\alpha$ is left-computable if there exists a total recursive function $g: \mathbb{N}^{+} \rightarrow \mathbb{Q}$ such that $g(n) \leq \alpha$ for all $n \in \mathbb{N}^{+}$and $\lim _{n \rightarrow \infty} g(n)=\alpha$. On the other hand, we say that a real number $\alpha$ is right-computable if $-\alpha$ is left-computable. The following (i) and (ii) then hold:

(i) A real number $\alpha$ is computable if and only if $\alpha$ is both left-computable and right-computable.

(ii) A real number $\alpha$ is right-computable if and only if the set $\{r \in \mathbb{Q} \mid \alpha<r\}$ is r.e.

See e.g. Weihrauch [22] for the detail of the treatment of the computability of real numbers.

\subsection{Algorithmic information theory}

In the following we concisely review some definitions and results of algorithmic information theory $[4,5,6]$. A computer is a partial recursive function $C:\{0,1\}^{*} \rightarrow\{0,1\}^{*}$ such that $\operatorname{dom} C$ is a prefix-free set. For each computer $C$ and each $s \in\{0,1\}^{*}, H_{C}(s)$ is defined by $H_{C}(s)=$ $\min \left\{|p| \mid p \in\{0,1\}^{*} \& C(p)=s\right\}$ (may be $\infty$ ). A computer $U$ is said to be optimal if for each computer $C$ there exists $d \in \mathbb{N}$ with the following property; if $C(p)$ is defined, then there is a $p^{\prime}$ for which $U\left(p^{\prime}\right)=C(p)$ and $\left|p^{\prime}\right| \leq|p|+d$. It is easy to see that there exists an optimal computer. Note that the class of optimal computers equals to the class of functions which are computed by universal self-delimiting Turing machines (see Chaitin [4] for the detail). We choose a particular optimal computer $U$ as the standard one for use, and define $H(s)$ as $H_{U}(s)$, which is referred to as the program-size complexity of $s$, the information content of $s$, or the Kolmogorov complexity of $s$ $[8,11,4]$. It follows that for every computer $C$ there exists $d \in \mathbb{N}$ such that, every $s \in\{0,1\}^{*}$,

$$
H(s) \leq H_{C}(s)+d
$$

Based on this we can show that there exists $c \in \mathbb{N}$ such that, for every $s \neq \lambda$,

$$
H(s) \leq|s|+2 \log _{2}|s|+c .
$$

An element of $\operatorname{dom} U$ is called a program for $U$.

Chaitin's halting probability $\Omega$ is defined by $\Omega=\sum_{p \in \operatorname{dom} U} 2^{-|p|}$. For any $\alpha \in \mathbb{R}$, we say that $\alpha$ is weakly Chaitin random if there exists $c \in \mathbb{N}$ such that $n-c \leq H\left(\alpha_{n}\right)$ for all $n \in \mathbb{N}^{+}[4,6]$. Then Chaitin [4] showed that $\Omega$ is weakly Chaitin random. For any $\alpha \in \mathbb{R}$, we say that $\alpha$ is Chaitin random if $\lim _{n \rightarrow \infty} H\left(\alpha_{n}\right)-n=\infty[4,6]$. It is then shown that, for every $\alpha \in \mathbb{R}$, $\alpha$ is weakly Chaitin random if and only if $\alpha$ is Chaitin random (see Chaitin [6] for the proof and historical detail). Thus $\Omega$ is Chaitin random. 


\subsection{Partial randomness}

In the works $[16,17]$, we generalized the notion of the randomness of a real number so that the degree of the randomness, which is often referred to as the partial randomness recently $[2,13,3]$, can be characterized by a real number $T$ with $0 \leq T \leq 1$ as follows.

Definition 2.1 (weak Chaitin $T$-randomness). Let $T \in \mathbb{R}$ with $T \geq 0$. For any $\alpha \in \mathbb{R}$, we say that $\alpha$ is weakly Chaitin T-random if there exists $c \in \mathbb{N}$ such that $T n-c \leq H\left(\alpha_{n}\right)$ for all $n \in \mathbb{N}^{+}$.

Definition 2.2 (T-compressibility). Let $T \in \mathbb{R}$ with $T \geq 0$. For any $\alpha \in \mathbb{R}$, we say that $\alpha$ is T-compressible if $H\left(\alpha_{n}\right) \leq T n+o(n)$, which is equivalent to $\lim _{\sup _{n \rightarrow \infty}} H\left(\alpha_{n}\right) / n \leq T$.

In the case of $T=1$, the weak Chaitin $T$-randomness results in the weak Chaitin randomness. For every $T \in[0,1]$ and every $\alpha \in \mathbb{R}$, if $\alpha$ is weakly Chaitin $T$-random and $T$-compressible, then

$$
\lim _{n \rightarrow \infty} \frac{H\left(\alpha_{n}\right)}{n}=T
$$

The left-hand side of (3) is referred to as the compression rate of a real number $\alpha$ in general. Note, however, that (3) does not necessarily imply that $\alpha$ is weakly Chaitin $T$-random. Thus, the notion of partial randomness is a stronger representation of compression rate.

Definition 2.3 (Chaitin $T$-randomness, Tadaki $[16,17])$. Let $T \in \mathbb{R}$ with $T \geq 0$. For any $\alpha \in \mathbb{R}$, we say that $\alpha$ is Chaitin T-random if $\lim _{n \rightarrow \infty} H\left(\alpha_{n}\right)-T n=\infty$.

In the case of $T=1$, the Chaitin $T$-randomness results in the Chaitin randomness. Obviously, for every $T \in[0,1]$ and every $\alpha \in \mathbb{R}$, if $\alpha$ is Chaitin $T$-random, then $\alpha$ is weakly Chaitin $T$-random. However, in 2005 Reimann and Stephan [13] showed that, in the case of $T<1$, the converse does not necessarily hold. This contrasts with the equivalence between the weak Chaitin randomness and the Chaitin randomness, each of which corresponds to the case of $T=1$. Recently, KjosHanssen [10] showed that the distinction between the weak Chaitin $T$-randomness and the Chaitin $T$-randomness has important applications to the research on the notion of $T$-capacitability and its related notions $[9,14]$.

\section{The previous results}

In this section, we review some results of the statistical mechanical interpretation of AIT, developed by our former work [19]. We first introduce the notion of thermodynamic quantities into AIT in the following manner.

In statistical mechanics, the partition function $Z_{\mathrm{sm}}(T)$, free energy $F_{\mathrm{sm}}(T)$, energy $E_{\mathrm{sm}}(T)$, entropy $S_{\mathrm{sm}}(T)$, and specific heat $C_{\mathrm{sm}}(T)$ at temperature $T$ are given as follows:

$$
\begin{array}{cc}
Z_{\mathrm{sm}}(T)=\sum_{x \in X} e^{-\frac{E_{x}}{k_{\mathrm{B}} T}}, & F_{\mathrm{sm}}(T)=-k_{\mathrm{B}} T \ln Z_{\mathrm{sm}}(T), \\
E_{\mathrm{sm}}(T)=\frac{1}{Z_{\mathrm{sm}}(T)} \sum_{x \in X} E_{x} e^{-\frac{E_{x}}{k_{\mathrm{B}} T}}, & S_{\mathrm{sm}}(T)=\frac{E_{\mathrm{sm}}(T)-F_{\mathrm{sm}}(T)}{T}, \\
C_{\mathrm{sm}}(T)=\frac{d}{d T} E_{\mathrm{sm}}(T), &
\end{array}
$$


where $X$ is a complete set of energy eigenstates of a quantum system and $E_{x}$ is the energy of an energy eigenstate $x$. The constant $k_{\mathrm{B}}$ is called the Boltzmann Constant, and the ln denotes the natural logarithm. ${ }^{2}$

We introduce the notion of thermodynamic quantities into AIT by performing Replacements 1 below for the thermodynamic quantities (4) in statistical mechanics.

\section{Replacements 1.}

(i) Replace the complete set $X$ of energy eigenstates $x$ by the set $\operatorname{dom} U$ of all programs $p$ for $U$.

(ii) Replace the energy $E_{x}$ of an energy eigenstate $x$ by the length $|p|$ of a program $p$.

(iii) Set the Boltzmann Constant $k_{\mathrm{B}}$ to $1 / \ln 2$.

For that purpose, we first choose a particular recursive enumeration $p_{1}, p_{2}, p_{3}, p_{4}, \ldots$ of the infinite r.e. set $\operatorname{dom} U$ as the standard one for use throughout the rest of this paper. ${ }^{3}$ Then, motivated by the formulae (4) and taking into account Replacements 1 , we introduce the notion of thermodynamic quantities into AIT as follows.

Definition 3.1 (thermodynamic quantities in AIT, [19]). Let $T$ be any real number with $T>0$.

(i) The partition function $Z(T)$ at temperature $T$ is defined as $\lim _{k \rightarrow \infty} Z_{k}(T)$ where

$$
Z_{k}(T)=\sum_{i=1}^{k} 2^{-\frac{\left|p_{i}\right|}{T}} .
$$

(ii) The free energy $F(T)$ at temperature $T$ is defined as $\lim _{k \rightarrow \infty} F_{k}(T)$ where

$$
F_{k}(T)=-T \log _{2} Z_{k}(T) .
$$

(iii) The energy $E(T)$ at temperature $T$ is defined as $\lim _{k \rightarrow \infty} E_{k}(T)$ where

$$
E_{k}(T)=\frac{1}{Z_{k}(T)} \sum_{i=1}^{k}\left|p_{i}\right| 2^{-\frac{\left|p_{i}\right|}{T}} .
$$

(iv) The statistical mechanical entropy $S(T)$ at temperature $T$ is defined as $\lim _{k \rightarrow \infty} S_{k}(T)$ where

$$
S_{k}(T)=\frac{E_{k}(T)-F_{k}(T)}{T} .
$$

(v) The specific heat $C(T)$ at temperature $T$ is defined as $\lim _{k \rightarrow \infty} C_{k}(T)$ where $C_{k}(T)=E_{k}^{\prime}(T)$, the derived function of $E_{k}(T)$.

\footnotetext{
${ }^{2}$ For the thermodynamic quantities in statistical mechanics, see e.g. Chapter 16 of [1] and Chapter 2 of [21]. To be precise, the partition function is not a thermodynamic quantity but a statistical mechanical quantity.

${ }^{3}$ Actually, the enumeration $\left\{p_{i}\right\}$ can be chosen quite arbitrarily, and the results of this paper are independent of the choice of $\left\{p_{i}\right\}$. This is because the sum $\sum_{i=1}^{k} 2^{-\left|p_{i}\right| / T}$ and $\sum_{i=1}^{k}\left|p_{i}\right| 2^{-\left|p_{i}\right| / T}$ in Definition 3.1 are positive term series and converge as $k \rightarrow \infty$ for every $T \in(0,1)$ (see Lemma 4.5 (i) below). For the sake of convenience, however, we choose $\left\{p_{i}\right\}$ to be a recursive enumeration of $\operatorname{dom} U$ in this paper.
} 
Note that $Z(1)=\Omega$ in particular. Then Theorems 3.2 and 3.3 below hold for these thermodynamic quantities in AIT.

Theorem 3.2 (properties of $Z(T)$ and $F(T),[16,17,19])$. Let $T \in \mathbb{R}$.

(i) If $0<T \leq 1$ and $T$ is computable, then each of $Z(T)$ and $F(T)$ converges and is weakly Chaitin $T$-random and $T$-compressible.

(ii) If $1<T$, then $Z(T)$ and $F(T)$ diverge to $\infty$ and $-\infty$, respectively.

Theorem 3.3 (properties of $E(T), S(T)$, and $C(T),[19])$. Let $T \in \mathbb{R}$.

(i) If $0<T<1$ and $T$ is computable, then each of $E(T), S(T)$, and $C(T)$ converges and is Chaitin T-random and T-compressible.

(ii) If $1 \leq T$, then both $E(T)$ and $S(T)$ diverge to $\infty$. In the case of $T=1, C(T)$ diverges to $\infty^{4}$

The above two theorems show that if $T$ is a computable real number with $T \in(0,1)$ then the temperature $T$ equals to the partial randomness (and therefore the compression rate) of the values of all the thermodynamic quantities in Definition 3.1. Note also that the weak Chaitin $T$-randomness of thermodynamic quantities for $T<1$ in Theorems 3.2 is strengthen to the Chaitin $T$-randomness in Theorem 3.3 in exchange for the divergence of thermodynamic quantities at $T=1$.

In statistical mechanics or thermodynamics, among all thermodynamic quantities one of the most typical thermodynamic quantities is temperature itself. Inspired by this fact in physics and the above observation on the role of the temperature $T$ in the statistical mechanical interpretation of AIT, the following question thus arises naturally: Can the partial randomness of the temperature $T$ equal to the temperature $T$ itself in the statistical mechanical interpretation of AIT ? This question is rather self-referential. However, we can answer it affirmatively in the following form.

Theorem 3.4 (fixed point theorem on partial randomness, [19]). For every $T \in(0,1)$, if $Z(T)$ is computable, then $T$ is weakly Chaitin $T$-random and $T$-compressible, and therefore

$$
\lim _{n \rightarrow \infty} \frac{H\left(T_{n}\right)}{n}=T
$$

Theorem 3.4 is just a fixed point theorem on partial randomness, where the computability of the value $Z(T)$ gives a sufficient condition for a real number $T \in(0,1)$ to be a fixed point on partial randomness. Thus, the above observation that the temperature $T$ equals to the partial randomness of the values of the thermodynamic quantities in the statistical mechanical interpretation of AIT is further confirmed. In this paper, we confirm this observation much further by showing that fixed point theorems of the same form as Theorem 3.4 hold also for free energy $F(T)$, energy $E(T)$, and statistical mechanical entropy $S(T)$. For completeness, we include the proof of Theorem 3.4 in Appendix A.

\footnotetext{
${ }^{4}$ It is still open whether $C(T)$ diverges or not in the case of $T>1$.
} 


\section{The main results}

The following three theorems are the main results of this paper.

Theorem 4.1 (fixed point theorem by free energy). For every $T \in(0,1)$, if $F(T)$ is computable then $T$ is weakly Chaitin T-random and T-compressible.

Theorem 4.2 (fixed point theorem by energy). For every $T \in(0,1)$, if $E(T)$ is computable then $T$ is Chaitin T-random and T-compressible.

Theorem 4.3 (fixed point theorem by statistical mechanical entropy). For every $T \in(0,1)$, if $S(T)$ is computable then $T$ is Chaitin $T$-random and $T$-compressible.

First, note that the weak Chaitin $T$-randomness of $T$ in Theorems 3.4 is strengthen to the Chaitin $T$-randomness of $T$ in Theorems 4.2 and 4.3 .

The proof of Theorem 4.1 uses Theorems 4.6, 4.8, and 4.9 below. On the other hand, the proofs of Theorems 4.2 and 4.3 use Theorems 4.7, 4.8, and 4.9 below. All these proofs also use Theorem 4.4 below, where the thermodynamic relations in statistical mechanics are recovered by the thermodynamic quantities of AIT. We complete the proofs of Theorems 4.1, 4.2, and 4.3 in the next section. Compared with the proof of Theorem 4.1, the proofs of Theorems 4.2 and 4.3 are more delicate.

Theorem 4.4 (thermodynamic relations).

(i) $F_{k}^{\prime}(T)=-S_{k}(T), E_{k}^{\prime}(T)=C_{k}(T)$, and $S_{k}^{\prime}(T)=C_{k}(T) / T$ for every $k \in \mathbb{N}^{+}$and every $T \in(0,1)$.

(ii) $F^{\prime}(T)=-S(T), E^{\prime}(T)=C(T)$, and $S^{\prime}(T)=C(T) / T$ for every $T \in(0,1)$.

(iii) $S_{k}(T), C_{k}(T) \geq 0$ for every $k \in \mathbb{N}^{+}$and every $T \in(0,1)$. There exists $k_{0} \in \mathbb{N}^{+}$such that, for every $k \geq k_{0}$ and every $T \in(0,1), S_{k}(T), C_{k}(T)>0$. Moreover, $S(T), C(T)>0$ for every $T \in(0,1)$.

The proof of Theorem 4.4 uses Lemma 4.5 below. For each $T \in(0,1)$, we define $W(T)$ and $Y(T)$ as $\lim _{k \rightarrow \infty} W_{k}(T)$ and $\lim _{k \rightarrow \infty} Y_{k}(T)$, respectively, where $W_{k}(T)=\sum_{i=1}^{k}\left|p_{i}\right| 2^{-\frac{\left|p_{i}\right|}{T}}$ and $Y_{k}(T)=$ $\sum_{i=1}^{k}\left|p_{i}\right|^{2} 2^{-\frac{\left|p_{i}\right|}{T}}$.

\section{Lemma 4.5.}

(i) For every $T \in(0,1)$, the limit values $Z(T), W(T)$, and $Y(T)$ exist, and are positive real numbers.

(ii) The sequence $\left\{Z_{k}(T)\right\}_{k}$ of functions of $T$ is uniformly convergent on $(0,1)$ in the wider sense. The same holds for the sequences $\left\{W_{k}(T)\right\}_{k}$ and $\left\{Y_{k}(T)\right\}_{k}$.

(iii) The function $Z(T)$ of $T$ is continuous on $(0,1)$. The same holds for the functions $W(T)$ and $Y(T)$. 
Proof. (i) Suppose that $T$ is an arbitrary real number with $T \in(0,1)$.

First, we show that $Y_{k}(T)$ converges as $k \rightarrow \infty$. Since $T<1$, there is $l_{0} \in \mathbb{N}^{+}$such that

$$
\frac{1}{T}-2 \frac{\log _{2} l}{l} \geq 1
$$

for all $l \geq l_{0}$. Then, since $\lim _{k \rightarrow \infty}\left|p_{k}\right|=\infty$, there is $k_{0} \in \mathbb{N}^{+}$such that $\left|p_{i}\right| \geq l_{0}$ for all $i>k_{0}$. Thus, we see that, for each $i>k_{0}$,

$$
\left|p_{i}\right|^{2} 2^{-\frac{\left|p_{i}\right|}{T}}=2^{-\left(\frac{1}{T}-2 \frac{\log _{2}\left|p_{i}\right|}{\left|p_{i}\right|}\right)\left|p_{i}\right|} \leq 2^{-\left|p_{i}\right|} .
$$

Hence, for each $k>k_{0}$,

$$
Y_{k}(T)-Y_{k_{0}}(T)=\sum_{i=k_{0}+1}^{k}\left|p_{i}\right|^{2} 2^{-\frac{\left|p_{i}\right|}{T}} \leq \sum_{i=k_{0}+1}^{k} 2^{-\left|p_{i}\right|}<\Omega=Z(1) .
$$

Therefore, since $\left\{Y_{k}(T)\right\}_{k}$ is an increasing sequence of positive real numbers bounded to the above, it converges to a positive real number as $k \rightarrow \infty$, as desired.

Note that $0<Z_{k}(T) \leq W_{k}(T) \leq Y_{k}(T)$ for every $k \in \mathbb{N}^{+}$, and the sequences $\left\{Z_{k}(T)\right\}_{k}$ and $\left\{W_{k}(T)\right\}_{k}$ of positive real numbers are increasing. It follows that $Z_{k}(T)$ and $W_{k}(T)$ converge to a positive real number as $k \rightarrow \infty$.

(ii) Note that, for every $k \in \mathbb{N}^{+}$and every $t, T \in(0,1)$ with $t \leq T$,

$$
0<Z(t)-Z_{k}(t)=\sum_{i=k+1}^{\infty} 2^{-\frac{\left|p_{i}\right|}{t}} \leq \sum_{i=k+1}^{\infty} 2^{-\frac{\left|p_{i}\right|}{T}}=Z(T)-Z_{k}(T) .
$$

It follows that the sequence $\left\{Z_{k}(T)\right\}_{k}$ of functions of $T$ is uniformly convergent on $(0,1)$ in the wider sense. In the same manner, we can show that the sequences $\left\{W_{k}(T)\right\}_{k}$ and $\left\{Y_{k}(T)\right\}_{k}$ are uniformly convergent on $(0,1)$ in the wider sense.

(iii) Note that, for each $k \in \mathbb{N}^{+}$, the mapping $(0,1) \ni T \mapsto Z_{k}(T)$ is a continuous function. It follows from Lemma 4.5 (ii) that the function $Z(T)$ of $T$ is continuous on $(0,1)$. In the same manner, we can show that the functions $W(T)$ and $Y(T)$ of $T$ are continuous on $(0,1)$.

The proof of Theorem 4.4 is then given as follows.

Proof of Theorem 4.4. (i) First, from Definition 3.1 we see that, for every $k \in \mathbb{N}^{+}$and every $T \in(0,1)$,

$$
\begin{aligned}
F_{k}(T) & =-T \log _{2} Z_{k}(T), \\
E_{k}(T) & =\frac{W_{k}(T)}{Z_{k}(T)}, \\
S_{k}(T) & =\frac{W_{k}(T)}{T Z_{k}(T)}+\log _{2} Z_{k}(T), \\
Z_{k}^{\prime}(T) & =\frac{\ln 2}{T^{2}} W_{k}(T), \\
W_{k}^{\prime}(T) & =\frac{\ln 2}{T^{2}} Y_{k}(T) .
\end{aligned}
$$


Thus, by straightforward differentiation, we can check that the relations of Theorem 4.4 (i) hold. For example, it follows from (7) and (6) that, for every $k \in \mathbb{N}^{+}$and every $T \in(0,1)$,

$$
S_{k}^{\prime}(T)=\frac{1}{T} E_{k}^{\prime}(T)-\frac{1}{T^{2}} \frac{W_{k}(T)}{Z_{k}(T)}+\frac{1}{\ln 2} \frac{Z_{k}^{\prime}(T)}{Z_{k}(T)} .
$$

Using the definition $C_{k}(T)=E_{k}^{\prime}(T)$ and the equation (8) we see that, for every $k \in \mathbb{N}^{+}$and every $T \in(0,1), S_{k}^{\prime}(T)=C_{k}(T) / T$.

(ii) From (6), (8), (9), and the definition $C_{k}(T)=E_{k}^{\prime}(T)$, we see that, for every $k \in \mathbb{N}^{+}$and every $T \in(0,1)$,

$$
C_{k}(T)=\frac{\ln 2}{T^{2}}\left\{\frac{Y_{k}(T)}{Z_{k}(T)}-\left(\frac{W_{k}(T)}{Z_{k}(T)}\right)^{2}\right\} .
$$

Thus, using Lemma 4.5 (i) above and the equations (5), (6), (7), and (10), we can first see that the limit values $F(T), E(T), S(T)$, and $C(T)$ exist for every $T \in(0,1)$. Using Lemma 4.5 in whole and the equations (7) and (10), we can next check that the sequences $\left\{-S_{k}(T)\right\}_{k},\left\{C_{k}(T)\right\}_{k}$ and $\left\{C_{k}(T) / T\right\}_{k}$ of functions of $T$ are uniformly convergent on $(0,1)$ in the wider sense. Hence, Theorem 4.4 (ii) follows immediately from Theorem 4.4 (i).

(iii) From (7) we see that, for every $k \in \mathbb{N}^{+}$and every $T \in(0,1)$,

$$
S_{k}(T)=-\sum_{i=1}^{k} \frac{2^{-\frac{\left|p_{i}\right|}{T}}}{Z_{k}(T)} \log _{2} \frac{2^{-\frac{\left|p_{i}\right|}{T}}}{Z_{k}(T)} .
$$

Thus, $S_{k}(T) \geq 0$ for every $k \in \mathbb{N}^{+}$. We also see that, for every $k \geq 2$ and every $T \in(0,1)$,

$$
S_{k}(T) \geq-\frac{2^{-\frac{\left|p_{1}\right|}{T}}}{Z_{k}(T)} \log _{2} \frac{2^{-\frac{\left|p_{1}\right|}{T}}}{Z_{k}(T)}>0 .
$$

Hence, for every $T \in(0,1)$,

$$
S(T) \geq-\frac{2^{-\frac{\left|p_{1}\right|}{T}}}{Z(T)} \log _{2} \frac{2^{-\frac{\left|p_{1}\right|}{T}}}{Z(T)}>0 .
$$

On the other hand, from (10) we see that, for every $k \in \mathbb{N}^{+}$and every $T \in(0,1)$,

$$
C_{k}(T)=\frac{\ln 2}{T^{2}} \sum_{i=1}^{k}\left\{\left|p_{i}\right|-E_{k}(T)\right\}^{2} \frac{2^{-\frac{\left|p_{i}\right|}{T}}}{Z_{k}(T)} .
$$

Thus, $C_{k}(T) \geq 0$ for every $k \in \mathbb{N}^{+}$and every $T \in(0,1)$. We note that there exists $l \in \mathbb{N}^{+}$such that $\left|p_{l}\right| \leq\left|p_{i}\right|$ for every $i \in \mathbb{N}^{+}$. It is then easy to see that there exists $k_{0} \in \mathbb{N}^{+}$such that, for every $k \geq k_{0}$ and every $T \in(0,1),\left|p_{l}\right|<E_{k}(T)$. This is because there exists $i \in \mathbb{N}^{+}$such that $\left|p_{l}\right|<\left|p_{i}\right|$. Thus, by (11) we see that, for every $k \geq \max \left\{l, k_{0}\right\}$ and every $T \in(0,1)$,

$$
C_{k}(T) \geq \frac{\ln 2}{T^{2}}\left\{\left|p_{l}\right|-E_{k}(T)\right\}^{2} \frac{2^{-\frac{\left|p_{l}\right|}{T}}}{Z_{k}(T)}>0 .
$$

It is also easy to see that $\left|p_{l}\right|<E(T)$ for every $T \in(0,1)$. It follows from (12) that $C(T)>0$ for every $T \in(0,1)$. 
Theorem 4.6. Let $f:(0,1) \rightarrow \mathbb{R}$. Suppose that $f$ is increasing and there exists $g:(0,1) \times \mathbb{N}^{+} \rightarrow \mathbb{R}$ which satisfies the following four conditions:

(i) $\lim _{k \rightarrow \infty} g(T, k)=f(T)$ for every $T \in(0,1)$.

(ii) $\left\{(q, r, k) \in \mathbb{Q} \times(\mathbb{Q} \cap(0,1)) \times \mathbb{N}^{+} \mid q<g(r, k)\right\}$ is an r.e. set.

(iii) For every $T \in(0,1)$, there exist $a \in \mathbb{N}, k_{0} \in \mathbb{N}^{+}$, and $t \in(T, 1)$ such that, for every $k \geq k_{0}$ and every $x \in(T, t), g(x, k)-g(T, k) \leq 2^{a}(x-T)$.

(iv) For every $T \in(0,1)$, there exist $b \in \mathbb{N}$ and $k_{1} \in \mathbb{N}^{+}$such that, for every $k \geq k_{1}$,

$$
2^{-\frac{\left|p_{k+1}\right|}{T}-b} \leq g(T, k+1)-g(T, k) .
$$

Then, for every $T \in(0,1)$, if $f(T)$ is right-computable then $T$ is weakly Chaitin $T$-random.

Proof. The proof of Theorem 4.6 is obtained by slightly simplifying the proof of Theorem 4.7 below.

Theorem 4.7. Let $f:(0,1) \rightarrow \mathbb{R}$. Suppose that $f$ is increasing and there exists $g:(0,1) \times \mathbb{N}^{+} \rightarrow \mathbb{R}$ which satisfies the following four conditions:

(i) $\lim _{k \rightarrow \infty} g(T, k)=f(T)$ for every $T \in(0,1)$.

(ii) $\left\{(q, r, k) \in \mathbb{Q} \times(\mathbb{Q} \cap(0,1)) \times \mathbb{N}^{+} \mid q<g(r, k)\right\}$ is an r.e. set.

(iii) For every $T \in(0,1)$, there exist $a \in \mathbb{N}, k_{0} \in \mathbb{N}^{+}$, and $t \in(T, 1)$ such that, for every $k \geq k_{0}$ and every $x \in(T, t), g(x, k)-g(T, k) \leq 2^{a}(x-T)$.

(iv) For every $T \in(0,1)$, there exist $b \in \mathbb{N}, c \in \mathbb{N}^{+}$, and $k_{1} \in \mathbb{N}^{+}$such that, for every $k \geq k_{1}$,

$$
\left|p_{k+1}\right|^{c} 2^{-\frac{\left|p_{k+1}\right|}{T}-b} \leq g(T, k+1)-g(T, k) .
$$

Then, for every $T \in(0,1)$, if $f(T)$ is right-computable then $T$ is Chaitin $T$-random.

Proof. Suppose that $T \in(0,1)$ and $f(T)$ is right-computable. Then there exists a total recursive function $h: \mathbb{N}^{+} \rightarrow \mathbb{Q}$ such that $f(T) \leq h(m)$ for all $m \in \mathbb{N}^{+}$and $\lim _{m \rightarrow \infty} h(m)=f(T)$.

Since the condition (iii) holds for $g$, there exist $a \in \mathbb{N}, k_{0} \in \mathbb{N}^{+}$, and $t \in(T, 1)$ such that

$$
g(x, k)-g(T, k) \leq 2^{a}(x-T)
$$

for every $k \geq k_{0}$ and every $x \in(T, t)$. We choose any one $n_{0} \in \mathbb{N}^{+}$such that $0 . T_{n}+2^{-n}<t$ for all $n \geq n_{0}$. Such $n_{0}$ exists since $T<t$ and $\lim _{n \rightarrow \infty} 0 . T_{n}+2^{-n}=T$. Since $T_{n}$ is the first $n$ bits of the base-two expansion of $T$ with infinitely many zeros, we further see that $T<0 . T_{n}+2^{-n}<t$ for all $n \geq n_{0}$.

On the other hand, since the condition (iv) holds for $g$, there exist $b \in \mathbb{N}, c \in \mathbb{N}^{+}$, and $k_{1} \in \mathbb{N}^{+}$ such that

$$
\left|p_{k+1}\right|{ }^{c} 2^{-\frac{\left|p_{k+1}\right|}{T}-b} \leq g(T, k+1)-g(T, k)
$$


for every $k \geq k_{1}$. Without loss of generality, we can assume that $k_{1}=k_{0}$. Thus, since $g(T, k)$ is increasing on $k$ with $k \geq k_{0}$ and the condition (i) holds,

$$
\left|p_{i}\right|^{c} 2^{-\frac{\left|p_{i}\right|}{T}-b}<f(T)-g(T, k)
$$

if $i>k \geq k_{0}$.

Now, given $T_{n}$ with $n \geq n_{0}$, one can effectively find $k_{e}, m_{e} \in \mathbb{N}^{+}$such that $k_{e} \geq k_{0}$ and $h\left(m_{e}\right)<g\left(0 . T_{n}+2^{-n}, k_{e}\right)$. This is possible because $f(T)<f\left(0 . T_{n}+2^{-n}\right), \lim _{k \rightarrow \infty} g\left(0 . T_{n}+2^{-n}, k\right)=$ $f\left(0 . T_{n}+2^{-n}\right)$, and the condition (ii) holds for $g$. It follows from $f(T) \leq h\left(m_{e}\right)$ and (13) that $f(T)-g\left(T, k_{e}\right)<g\left(0 . T_{n}+2^{-n}, k_{e}\right)-g\left(T, k_{e}\right) \leq 2^{a-n}$. It follows from (14) that, for every $i>k_{e}$, $\left|p_{i}\right|^{c} 2^{-\frac{\left|p_{i}\right|}{T}-b}<2^{a-n}$ and therefore $c T \log _{2}\left|p_{i}\right|-(a+b) T<\left|p_{i}\right|-T n$. Thus, by calculating the set $\left\{U\left(p_{i}\right) \mid i \leq k_{e}\right\}$ and picking any one finite binary string $s$ which is not in this set, one can then obtain $s \in\{0,1\}^{*}$ such that $c T \log _{2} H(s)-(a+b) T<H(s)-T n$.

Hence, there exists a partial recursive function $\Psi:\{0,1\}^{*} \rightarrow\{0,1\}^{*}$ such that

$$
c T \log _{2} H\left(\Psi\left(T_{n}\right)\right)-(a+b) T<H\left(\Psi\left(T_{n}\right)\right)-T n
$$

for all $n \geq n_{0}$. Applying this inequality to itself, we have $c T \log _{2} n<H\left(\Psi\left(T_{n}\right)\right)-T n+O(1)$, for all $n \in \mathbb{N}^{+}$. On the other hand, using (1), there is $c_{\Psi} \in \mathbb{N}$ such that $H\left(\Psi\left(T_{n}\right)\right) \leq H\left(T_{n}\right)+c_{\Psi}$ for all $n \geq n_{0}$. It follows that $c T \log _{2} n<H\left(T_{n}\right)-T n+O(1)$. Hence, $T$ is Chaitin $T$-random.

Theorem 4.8. Let $f:(0,1) \rightarrow \mathbb{R}$. Suppose that $f$ is increasing and there exists $g:(0,1) \times \mathbb{N}^{+} \rightarrow \mathbb{R}$ which satisfies the following three conditions:

(i) For every $T \in(0,1), \lim _{k \rightarrow \infty} g(T, k)=f(T)$.

(ii) For every $T_{1}, T_{2} \in(0,1)$ with $T_{1}<T_{2}$, there exists $k_{0} \in \mathbb{N}^{+}$such that, for every $k \geq k_{0}$ and every $x \in\left[T_{1}, T_{2}\right], g(x, k) \leq f(x)$.

(iii) The set $\left\{(q, r, k) \in \mathbb{Q} \times(\mathbb{Q} \cap(0,1)) \times \mathbb{N}^{+} \mid q<g(r, k)\right\}$ is r.e.

Then, for every $T \in(0,1)$, if $f(T)$ is right-computable then $T$ is also right-computable.

Proof. Suppose that $T \in(0,1)$. We choose any $t_{1}, t_{2} \in \mathbb{Q}$ with $0<t_{1}<T<t_{2}<1$. Then, since the condition (ii) holds for $g$, there exists $k_{0} \in \mathbb{N}^{+}$such that $g(x, k) \leq f(x)$ for every $k \geq k_{0}$ and every $x \in\left[t_{1}, t_{2}\right]$. Suppose further that $f(T)$ is right-computable. Then there exists a total recursive function $h: \mathbb{N}^{+} \rightarrow \mathbb{Q}$ such that $f(T) \leq h(m)$ for all $m \in \mathbb{N}^{+}$and $\lim _{m \rightarrow \infty} h(m)=f(T)$. Thus, since $f$ is increasing and the condition (i) holds for $g$, we see that, for every $r \in \mathbb{Q} \cap\left[t_{1}, t_{2}\right]$, $T<r$ if and only if $\exists m \exists k \geq k_{0} h(m)<g(r, k)$. Since the condition (iii) holds for $g$, the set $\left\{r \in \mathbb{Q} \cap\left[t_{1}, t_{2}\right] \mid \exists m \exists k \geq k_{0} h(m)<g(r, k)\right\}$ is r.e. and therefore the set $\left\{r \in \mathbb{Q} \cap\left[t_{1}, t_{2}\right] \mid T<r\right\}$ is r.e. It follows from $T \in\left(t_{1}, t_{2}\right)$ that $T$ is right-computable.

Theorem 4.9. Let $f:(0,1) \rightarrow \mathbb{R}$. Suppose that there exists $g:(0,1) \times \mathbb{N}^{+} \rightarrow \mathbb{R}$ which satisfies the following six conditions:

(i) For every $T \in(0,1), \lim _{k \rightarrow \infty} g(T, k)=f(T)$.

(ii) For every $T \in(0,1)$, there exists $k_{0} \in \mathbb{N}^{+}$such that, for every $k \geq k_{0}, g(T, k)<f(T)$. 
(iii) For every $T \in(0,1)$, there exist $a \in \mathbb{N}, k_{1} \in \mathbb{N}^{+}$, and $t \in(T, 1)$ such that, for every $k \geq k_{1}$ and every $x \in(T, t), g(x, k)-g(T, k) \geq 2^{-a}(x-T)$.

(iv) For every $T \in(0,1)$, there exist $b \in \mathbb{N}, c \in \mathbb{N}$, and $k_{2} \in \mathbb{N}^{+}$such that, for every $k \geq k_{2}$,

$$
g(T, k+1)-g(T, k) \leq\left|p_{k+1}\right|^{b} 2^{-\left|p_{k+1}\right| / T+c} .
$$

(v) For each $k \in \mathbb{N}^{+}$, the mapping $(0,1) \ni T \mapsto g(T, k)$ is a continuous function.

(vi) $\left\{(q, r, k) \in \mathbb{Q} \times(\mathbb{Q} \cap(0,1)) \times \mathbb{N}^{+} \mid q>g(r, k)\right\}$ is an r.e. set.

Then, for every $T \in(0,1)$, if $f(T)$ is left-computable and $T$ is right-computable, then $T$ is $T$ compressible.

Proof. Suppose that $T \in(0,1)$. Since the condition (ii) holds for $g$, there exists $k_{0} \in \mathbb{N}^{+}$such that

$$
g(T, k)<f(T)
$$

for every $k \geq k_{0}$. Since the condition (iii) holds for $g$, there exist $a \in \mathbb{N}, k_{1} \in \mathbb{N}^{+}$, and $t \in(T, 1$ ) such that

$$
g(x, k)-g(T, k) \geq 2^{-a}(x-T)
$$

for every $k \geq k_{1}$ and every $x \in(T, t)$. Since the condition (iv) holds for $g$, there exist $b \in \mathbb{N}, c \in \mathbb{N}$, and $k_{2} \in \mathbb{N}^{+}$such that

$$
g(T, k+1)-g(T, k) \leq\left|p_{k+1}\right|^{b} 2^{-\left|p_{k+1}\right| / T+c}
$$

for every $k \geq k_{2}$. Without loss of generality, we can assume that $k_{0}=k_{1}=k_{2}$.

Suppose further that $T$ is right-computable and $f(T)$ is left-computable. Then there exists a total recursive function $A: \mathbb{N}^{+} \rightarrow \mathbb{Q}$ such that $T<A(l)<t$ for all $l \in \mathbb{N}^{+}$and $\lim _{l \rightarrow \infty} A(l)=T$, and there exists a total recursive function $B: \mathbb{N}^{+} \rightarrow \mathbb{Q}$ such that $B(m) \leq f(T)$ for all $m \in \mathbb{N}^{+}$and $\lim _{m \rightarrow \infty} B(m)=f(T)$.

Let $u$ be an arbitrary computable real number with $T<u<1$, and let $\beta=\sum_{i=1}^{\infty}\left|p_{i}\right|^{b} 2^{-\left|p_{i}\right| / u}$. Note that this limit exists and is weakly Chaitin $u$-random (see Theorem 3.2 (a) of [17] and Theorem 3 (i) of [19]). Thus, the base-two expansion of $\beta$ contains infinitely many zeros and infinitely many ones.

Given $n$ and $\beta_{\lceil T n / u\rceil}$ (i.e., the first $\lceil T n / u\rceil$ bits of the base-two expansion of $\beta-\lfloor\beta\rfloor$ ), one can effectively find $k_{e} \in \mathbb{N}^{+}$such that $k_{e} \geq k_{0}$ and

$$
0 . \beta_{\lceil T n / u\rceil}+\lfloor\beta\rfloor<\sum_{i=1}^{k_{e}}\left|p_{i}\right|^{b} 2^{-\frac{\left|p_{i}\right|}{u}} .
$$

This is possible since $0 . \beta_{\lceil T n / u\rceil}+\lfloor\beta\rfloor<\beta$ and $\lim _{k \rightarrow \infty} \sum_{i=1}^{k}\left|p_{i}\right|^{b} 2^{-\left|p_{i}\right| / u}=\beta$. Since $\beta-\left(0 . \beta_{\lceil T n / u\rceil}+\right.$ $\lfloor\beta\rfloor) \leq 2^{-\lceil T n / u\rceil} \leq 2^{-T n / u}$, it is then shown that

$$
\sum_{i=k_{e}+1}^{\infty}\left|p_{i}\right|^{b} 2^{-\frac{\left|p_{i}\right|}{u}}=\beta-\sum_{i=1}^{k_{e}}\left|p_{i}\right|^{b} 2^{-\frac{\left|p_{i}\right|}{u}}<2^{-T n / u} .
$$


Raising both ends of this inequality to the power $u / T$ and using the inequality $x^{z}+y^{z} \leq(x+y)^{z}$ for real numbers $x, y>0$ and $z \geq 1$, we have

$$
\sum_{i=k_{e}+1}^{\infty}\left|p_{i}\right|^{b} 2^{-\frac{\left|p_{i}\right|}{T}} \leq \sum_{i=k_{e}+1}^{\infty}\left|p_{i}\right|^{\frac{b u}{T}} 2^{-\frac{\left|p_{i}\right|}{T}}<2^{-n} .
$$

Using (17) and the condition (i), it follows that

$$
f(T)-g\left(T, k_{e}\right)<\sum_{i=k_{e}+1}^{\infty}\left|p_{i}\right|^{b} 2^{-\frac{\left|p_{i}\right|}{T}+c}<2^{c-n} .
$$

On the other hand, since the condition (v) holds for $g, g\left(T, k_{e}\right)=\lim _{l \rightarrow \infty} g\left(A(l), k_{e}\right)$. Obviously, $g\left(T, k_{e}\right)<f(T)$ by (15). Thus, since the condition (vi) holds for $g$, one can then effectively find $l_{e}, m_{e} \in \mathbb{N}^{+}$such that $g\left(A\left(l_{e}\right), k_{e}\right)<B\left(m_{e}\right)$. It follows from (18) and (16) that

$$
2^{c-n}>f(T)-g\left(T, k_{e}\right) \geq B\left(m_{e}\right)-g\left(T, k_{e}\right)>g\left(A\left(l_{e}\right), k_{e}\right)-g\left(T, k_{e}\right) \geq 2^{-a}\left(A\left(l_{e}\right)-T\right) .
$$

Thus, $0<A\left(l_{e}\right)-T<2^{a+c-n}$. Let $r_{n}$ be the first $n$ bits of the base-two expansion of the rational number $A\left(l_{e}\right)$ with infinitely many zeros. Then $\left|A\left(l_{e}\right)-0 . r_{n}\right|<2^{-n}$. It follows from $\left|T-0 . T_{n}\right|<$ $2^{-n}$ that $\left|0 . T_{n}-0 . r_{n}\right|<\left(2^{a+c}+2\right) 2^{-n}$. Hence, $T_{n}=r_{n}, r_{n} \pm 1, r_{n} \pm 2, \ldots, r_{n} \pm\left(2^{a+c}+1\right)$, where $T_{n}$ and $r_{n}$ are regarded as a dyadic integer. Thus, there are still $2^{a+c+1}+3$ possibilities of $T_{n}$, so that one needs only $a+c+3$ bits more in order to determine $T_{n}$.

Thus, there exists a partial recursive function $\Phi: \mathbb{N}^{+} \times\{0,1\}^{*} \times\{0,1\}^{*} \rightarrow\{0,1\}^{*}$ such that, for every $n \in \mathbb{N}^{+}$, there exists $s \in\{0,1\}^{*}$ with the properties that $|s|=a+c+3$ and $\Phi\left(n, \beta_{\lceil T n / u\rceil}, s\right)=$ $T_{n}$. It follows from (2) that $H\left(T_{n}\right) \leq\left|\beta_{\lceil T n / u\rceil}\right|+o(n) \leq T n / u+o(n)$, which implies that $T$ is $T / u$-compressible. Since $u$ is an arbitrary computable real number with $T<u<1$, it follows that $T$ is $T$-compressible.

\section{The proofs of the main results}

In this section we complete the proofs of our main results; Theorems 4.1, 4.2, and 4.3.

\subsection{The proof of Theorem 4.1}

We first complete the proof of Theorem 4.1, based on Theorems 4.4, 4.6, 4.8, and 4.9, as follows.

Let $f:(0,1) \rightarrow \mathbb{R}$ with $f(T)=-F(T)$, and let $g:(0,1) \times \mathbb{N}^{+} \rightarrow \mathbb{R}$ with $g(T, k)=-F_{k}(T)$. First, it follows from Theorem 4.4 (ii) and (iii) that $f$ is increasing.

Obviously, $\lim _{k \rightarrow \infty} g(T, k)=f(T)$ for every $T \in(0,1)$. Using the mean value theorem we see that, for every $T \in(0,1)$ and every $k \in \mathbb{N}^{+}$,

$$
\frac{2^{-\frac{\left|p_{k+1}\right|}{T}}}{Z_{k+1}(T)}<\ln Z_{k+1}(T)-\ln Z_{k}(T)<\frac{2^{-\frac{\left|p_{k+1}\right|}{T}}}{Z_{k}(T)} .
$$

It follows that, for every $T \in(0,1)$ and every $k \in \mathbb{N}^{+}, g(T, k)<g(T, k+1)$ and therefore $g(T, k)<$ $f(T)$. At this point, the conditions (i) and (ii) of Theorem 4.6, all conditions of Theorem 4.8, and the conditions (i), (ii), (v), and (vi) of Theorem 4.9 hold for $g$. 
Using (19) we see that, for every $T \in(0,1)$ and every $k \in \mathbb{N}^{+}$,

$$
\frac{T 2^{-\frac{\left|p_{k+1}\right|}{T}}}{Z_{k+1}(T) \ln 2}<g(T, k+1)-g(T, k)<\frac{T 2^{-\frac{\left|p_{k+1}\right|}{T}}}{Z_{k}(T) \ln 2} .
$$

Thus, the condition (iv) of Theorem 4.6 and the condition (iv) of Theorem 4.9 hold for $g$.

Using the mean value theorem and Theorem 4.4 (i) and (iii), we see that

$$
S_{k}(T)(x-T) \leq g(x, k)-g(T, k) \leq S_{k}(t)(x-T)
$$

for every $k \in \mathbb{N}^{+}$and every $T, x, t \in(0,1)$ with $T<x<t$. On the other hand, we see that, for every $k \in \mathbb{N}^{+}$and every $T \in(0,1)$,

$$
E_{k+1}(T)-E_{k}(T)=\frac{Z_{k}(T)\left|p_{k+1}\right|-W_{k}(T)}{Z_{k+1}(T) Z_{k}(T)} 2^{-\frac{\left|p_{k+1}\right|}{T}}
$$

Recall here that, for every $T \in(0,1), \lim _{k \rightarrow \infty} Z_{k}(T)$ and $\lim _{k \rightarrow \infty} W_{k}(T)$ exist and are positive by Lemma 4.5 (i). It follows from $\lim _{k \rightarrow \infty}\left|p_{k+1}\right|=\infty$ that, for every $T \in(0,1)$, there exists $k_{0} \in \mathbb{N}^{+}$such that, for every $k \geq k_{0}, E_{k}(T)<E_{k+1}(T)$ and therefore $S_{k}(T)<S_{k+1}(T)$ by (19). Using Theorem 4.4 (iii), we see that, for every $T \in(0,1)$, there exists $k_{1} \in \mathbb{N}^{+}$such that, for every $k \geq k_{1}, 0<S_{k_{1}}(T) \leq S_{k}(T)<S(T)$. Thus, using (20), for every $T, t \in(0,1)$ with $T<t$, there exists $k_{2} \in \mathbb{N}^{+}$such that $S_{k_{2}}(T)>0$ and for every $k \geq k_{2}$ and every $x \in(T, t)$, $S_{k_{2}}(T)(x-T) \leq g(x, k)-g(T, k)<S(t)(x-T)$. Therefore, the condition (iii) of Theorem 4.6 and the condition (iii) of Theorem 4.9 hold for $g$.

Thus, Theorem 4.6, Theorem 4.8, and Theorem 4.9 result in the following three theorems, respectively.

Theorem 5.1. For every $T \in(0,1)$, if $F(T)$ is left-computable then $T$ is weakly Chaitin $T$ random.

Theorem 5.2. For every $T \in(0,1)$, if $F(T)$ is left-computable then $T$ is right-computable.

Theorem 5.3. For every $T \in(0,1)$, if both $F(T)$ and $T$ are right-computable then $T$ is $T$ compressible.

Theorem 4.1 follows immediately from these three theorems.

\subsection{The proof of Theorem 4.2}

We complete the proof of Theorem 4.2, based on Theorems 4.4, 4.7, 4.8, and 4.9, as follows.

Let $f:(0,1) \rightarrow \mathbb{R}$ with $f(T)=E(T)$, and let $g:(0,1) \times \mathbb{N}^{+} \rightarrow \mathbb{R}$ with $g(T, k)=E_{k}(T)$. First, by Theorem 4.4 (ii) and (iii), we see that $E^{\prime}(T)=C(T)>0$ for every $T \in(0,1)$. Thus $f$ is increasing.

Obviously, $\lim _{k \rightarrow \infty} g(T, k)=f(T)$ for every $T \in(0,1)$. At this point, the conditions (i) and (ii) of Theorem 4.7, the conditions (i) and (iii) of Theorem 4.8, and the conditions (i), (v), and (vi) of Theorem 4.9 hold for $g$.

We see that, for every $k \in \mathbb{N}^{+}$and every $T \in(0,1)$,

$$
E_{k+1}(T)-E_{k}(T)=\frac{Z_{k}(T)\left|p_{k+1}\right|-W_{k}(T)}{Z_{k+1}(T) Z_{k}(T)} 2^{-\frac{\left|p_{k+1}\right|}{T}} .
$$


Recall here that, for every $T \in(0,1), \lim _{k \rightarrow \infty} Z_{k}(T)$ and $\lim _{k \rightarrow \infty} W_{k}(T)$ exist and are positive by Lemma 4.5 (i). It follows from $\lim _{k \rightarrow \infty}\left|p_{k+1}\right|=\infty$ that, for every $T \in(0,1)$, there exist $a \in \mathbb{N}$, $b \in \mathbb{N}$, and $k_{0} \in \mathbb{N}^{+}$such that, every $k \geq k_{0}$,

$$
\left|p_{k+1}\right| 2^{-\frac{\left|p_{k+1}\right|}{T}-a} \leq g(T, k+1)-g(T, k) \leq\left|p_{k+1}\right| 2^{-\frac{\left|p_{k+1}\right|}{T}+b} .
$$

Thus, the condition (iv) of Theorem 4.7 and the condition (iv) of Theorem 4.9 hold for $g$. It follows from (22) that, for every $T \in(0,1)$, there exists $k_{0} \in \mathbb{N}^{+}$such that, every $k \geq k_{0}, g(T, k)<$ $g(T, k+1)$ and therefore $g(T, k)<f(T)$. Thus, the condition (ii) of Theorem 4.9 holds for $g$.

Using Lemma 4.5 (ii) and (iii) in addition to Lemma 4.5 (i), we can show a stronger statement than the inequalities (22). The stronger statement for the lower bound of (22) is needed here. That is, based on (21), Lemma 4.5, and $\lim _{k \rightarrow \infty}\left|p_{k+1}\right|=\infty$, we can show that, for every $T_{1}, T_{2} \in(0,1)$ with $T_{1}<T_{2}$, there exist $a \in \mathbb{N}$ and $k_{0} \in \mathbb{N}^{+}$such that, every $k \geq k_{0}$ and every $x \in\left[T_{1}, T_{2}\right]$,

$$
\left|p_{k+1}\right| 2^{-\frac{\left|p_{k+1}\right|}{x}-a} \leq g(x, k+1)-g(x, k) .
$$

It follows that the condition (ii) of Theorem 4.8 holds for $g$.

Now, using the mean value theorem and Theorem 4.4 (i), we see that, for every $k \in \mathbb{N}^{+}$and every $T, x, t \in(0,1)$ with $T<x<t$, there exists $y \in(T, x)$ such that

$$
g(x, k)-g(T, k)=C_{k}(y)(x-T) .
$$

On the other hand, using (10) we see that, for every $k \in \mathbb{N}^{+}$and every $T \in(0,1), C_{k+1}(T)-C_{k}(T)$ is calculated as

$$
\begin{aligned}
\frac{\ln 2}{T^{2}} \frac{2^{-\frac{\left|p_{k+1}\right|}{Z_{k+1}}(T)}}{Z_{k+1}}\left[\mid p_{k+1}\right. & -\left\{\frac{W_{k+1}(T)}{Z_{k+1}(T)}+\frac{W_{k}(T)}{Z_{k}(T)}\right\}\left|p_{k+1}\right| \\
& \left.+\left\{\frac{W_{k+1}(T)}{Z_{k+1}(T)}+\frac{W_{k}(T)}{Z_{k}(T)}\right\} \frac{W_{k}(T)}{Z_{k}(T)}-\frac{Y_{k}(T)}{Z_{k}(T)}\right] .
\end{aligned}
$$

Thus, based on Lemma 4.5 and $\lim _{k \rightarrow \infty}\left|p_{k+1}\right|=\infty$, we can show that, for every $T_{1}, T_{2} \in(0,1)$ with $T_{1}<T_{2}$, there exist $a \in \mathbb{N}$ and $k_{0} \in \mathbb{N}^{+}$such that, every $k \geq k_{0}$ and every $y \in\left[T_{1}, T_{2}\right]$,

$$
\left|p_{k+1}\right|^{2} 2^{-\frac{\left|p_{k+1}\right|}{y}-a} \leq C_{k+1}(y)-C_{k}(y) .
$$

It follows from Theorem 4.4 (iii) that, for every $T_{1}, T_{2} \in(0,1)$ with $T_{1}<T_{2}$, there exist $a \in \mathbb{N}$ and $k_{0} \in \mathbb{N}^{+}$such that, every $k \geq k_{0}$ and every $y \in\left[T_{1}, T_{2}\right]$,

$$
0<\min C_{k_{0}}\left(\left[T_{1}, T_{2}\right]\right) \leq C_{k}(y)<\max C\left(\left[T_{1}, T_{2}\right]\right)
$$

where $\min C_{k_{0}}\left(\left[T_{1}, T_{2}\right]\right)=\min \left\{C_{k_{0}}(z) \mid z \in\left[T_{1}, T_{2}\right]\right\}$ and $\max C\left(\left[T_{1}, T_{2}\right]\right)=\max \{C(z) \mid z \in$ $\left.\left[T_{1}, T_{2}\right]\right\}$. In particular, $\max C\left(\left[T_{1}, T_{2}\right]\right)$ exists. This is because the function $C(T)$ of $T$ is continuous on $(0,1)$ by Lemma 4.5 and $(10)$. It follows from $(23)$ and $(24)$ that, for every $T, t \in(0,1)$ with $T<t$, there exist $a \in \mathbb{N}, b \in \mathbb{N}$, and $k_{0} \in \mathbb{N}^{+}$such that, for every $k \geq k_{0}$ and every $x \in(T, t)$, $2^{-a}(x-T) \leq g(x, k)-g(T, k)<2^{b}(x-T)$. Therefore, the condition (iii) of Theorem 4.7 and the condition (iii) of Theorem 4.9 hold for $g$.

Thus, Theorem 4.7, Theorem 4.8, and Theorem 4.9 result in the following three theorems, respectively. 
Theorem 5.4. For every $T \in(0,1)$, if $E(T)$ is right-computable then $T$ is Chaitin T-random.

Theorem 5.5. For every $T \in(0,1)$, if $E(T)$ is right-computable then $T$ is also right-computable.

Theorem 5.6. For every $T \in(0,1)$, if $E(T)$ is left-computable and $T$ is right-computable, then $T$ is T-compressible.

Theorem 4.2 follows immediately from these three theorems.

\subsection{The proof of Theorem 4.3}

In a similar manner to the proof of Theorem 4.2 described in the previous subsection, we can prove Theorem 4.3, based on Theorems 4.4, 4.7, 4.8, and 4.9. It is easy to convert the proof of Theorem 4.2 into the the proof of Theorem 4.3 , because of the similarity between $E_{k}^{\prime}(T)=C_{k}(T)$ and $S_{k}^{\prime}(T)=C_{k}(T) / T$ given in Theorem 4.4 (i).

\section{Some properties of the sufficient conditions}

In this section, we investigate some properties of the sufficient conditions for $T$ to be a fixed point in the fixed point theorems on partial randomness; Theorems 3.4, 4.1, 4.2, and 4.3. First we show Theorems $6.1,6.2,6.3$, and 6.4 below on the sufficient conditions.

Theorem $6.1([19])$. The set $\{T \in(0,1) \mid Z(T)$ is computable $\}$ is dense in $(0,1)$.

Proof. Since the function $2^{-l / T}$ of $T$ is increasing on $(0,1)$ for each $l \in \mathbb{N}^{+}$, the function $Z(T)$ of $T$ is increasing on $(0,1)$. On the other hand, the function $Z(T)$ of $T$ is continuous on $(0,1)$ by Lemma 4.5 (iii). Thus, since the set of all computable real numbers is dense in $\mathbb{R}$, the result follows.

Theorem 6.2. The set $\{T \in(0,1) \mid F(T)$ is computable $\}$ is dense in $(0,1)$.

Proof. It follows from Theorem 4.4 (ii) and (iii) that the function $F(T)$ of $T$ is a decreasing continuous function on $(0,1)$. Since the set of all computable real numbers is dense in $\mathbb{R}$, the result follows.

In the same manner as the proof of Theorem 6.2, we can prove the following theorems for each of $E(T)$ and $S(T)$.

Theorem 6.3. The set $\{T \in(0,1) \mid E(T)$ is computable $\}$ is dense in $(0,1)$.

Theorem 6.4. The set $\{T \in(0,1) \mid S(T)$ is computable $\}$ is dense in $(0,1)$.

Since the relation $F(T)=-T \log _{2} Z(T)$ holds from Definition 3.1, we can show the following theorem on the relation between the sufficient conditions in the fixed point theorems by $Z(T)$ and $F(T)$.

Theorem 6.5. There does not exist $T \in(0,1)$ such that both $Z(T)$ and $F(T)$ are computable. 
Proof. Contrarily, assume that both $Z(T)$ and $F(T)$ are computable for some $T \in(0,1)$. Since $F(T)=-T \log _{2} Z(T)$ and $0<Z(T)<1$, it is easy to see that $T$ is computable. It follows from Theorem $3.2(\mathrm{i})$ that $Z(T)$ is weakly Chaitin $T$-random. However, this contradicts the assumption that $Z(T)$ is computable, and the result follows.

Thus, the computability of $F(T)$ gives completely different fixed points from the computability of $Z(T)$. This implies that neither the computability of $Z(T)$ nor the computability of $F(T)$ is the necessary condition for $T$ to be a fixed point on partial randomness at all.

In a similar manner, we can prove the following two theorems using the relations $S(T)=$ $E(T) / T+\log _{2} Z(T)$ and $S(T)=(E(T)-F(T)) / T$, respectively.

Theorem 6.6. There does not exist $T \in(0,1)$ such that $Z(T), E(T)$, and $S(T)$ are all computable.

Theorem 6.7. There does not exist $T \in(0,1)$ such that $F(T), E(T)$, and $S(T)$ are all computable.

Using the property of a fixed point in the fixed point theorems, we can show the following theorem.

Theorem 6.8. $S_{a} \cap S_{b}=\emptyset$ for any distinct computable real numbers $a, b \in(0,1]$, where $S_{a}=\{T \in$ $(0,1) \mid Z(a T)$ is computable $\}$.

Proof. Let $T \in(0,1)$, and let $a$ be a computable real number with $a \in(0,1]$. Suppose that $Z(a T)$ is computable. Then, by Theorem $3.4, \lim _{n \rightarrow \infty} H\left((a T)_{n}\right) / n=a T$. Note that $H\left((a T)_{n}\right)=$ $H\left(T_{n}\right)+O(1)$ for all $n \in \mathbb{N}^{+}$. It follows that $\lim _{n \rightarrow \infty} H\left(T_{n}\right) / n=a T$.

Thus, for every computable real numbers $a, b \in(0,1]$, if $S_{a} \cap S_{b} \neq \emptyset$ then $a=b$.

As a corollary of Theorem 6.8, we have the following, for example.

Corollary 6.9. For every $T \in(0,1)$, if $Z(T)$ is computable, then $Z(T / n)$ is not computable for every $n \in \mathbb{N}^{+}$with $n \geq 2$. In other words, for every $T \in(0,1)$, if the sum $\sum_{i=1}^{\infty} 2^{-\left|p_{i}\right| / T}$ is computable, then the corresponding power sum $\sum_{i=1}^{\infty}\left(2^{-\left|p_{i}\right| / T}\right)^{n}$ is not computable for every $n \in \mathbb{N}^{+}$ with $n \geq 2$.

\section{Acknowledgments}

This work was supported by CREST of the Japan Science and Technology Agency, by KAKENHI, Grant-in-Aid for Scientific Research (C) (20540134), and by SCOPE of the Ministry of Internal Affairs and Communications of Japan.

\section{References}

[1] H. B. Callen, Thermodynamics and an Introduction to Thermostatistics, 2nd ed. John Wiley \& Sons, Inc., Singapore, 1985.

[2] C. S. Calude, L. Staiger, and S. A. Terwijn, "On partial randomness," Ann. Pure Appl. Logic, vol. 138, pp. 20-30, 2006. 
[3] C. S. Calude and M. A. Stay, "Natural halting probabilities, partial randomness, and zeta functions," Inform. and Comput., vol. 204, pp. 1718-1739, 2006.

[4] G. J. Chaitin, "A theory of program size formally identical to information theory," J. Assoc. Comput. Mach., vol. 22, pp. 329-340, 1975.

[5] G. J. Chaitin, "Incompleteness theorems for random reals," Adv. in Appl. Math., vol. 8, pp. 119-146, 1987.

[6] G. J. Chaitin, Algorithmic Information Theory. Cambridge University Press, Cambridge, 1987.

[7] R. G. Downey and D. R. Hirschfeldt, Algorithmic randomness and complexity. Springer-Verlag, To appear.

[8] P. Gács, "On the symmetry of algorithmic information," Soviet Math. Dokl., vol. 15, pp. 14771480, 1974; correction, ibid. vol. 15, pp. 1480, 1974.

[9] B. Kjos-Hanssen, "Infinite subsets of random sets of integers," Math. Res. Lett., vol. 16, no. 1, pp. 103-110, 2009.

[10] B. Kjos-Hanssen, Private communication, September 2008.

[11] L. A. Levin, "Laws of information conservation (non-growth) and aspects of the foundations of probability theory," Problems of Inform. Transmission, vol. 10, pp. 206-210, 1974.

[12] F. Reif, Fundamentals of Statistical and Thermal Physics. McGraw-Hill, Inc., Singapore, 1965.

[13] J. Reimann and F. Stephan, On hierarchies of randomness tests. Proceedings of the 9th Asian Logic Conference, World Scientific Publishing, August 16-19, 2005, Novosibirsk, Russia.

[14] J. Reimann, "Effectively closed sets of measures and randomness," To appear in Ann. Pure Appl. Logic.

[15] D. Ruelle, Statistical Mechanics, Rigorous Results, 3rd ed. Imperial College Press and World Scientific Publishing Co. Pte. Ltd., Singapore, 1999.

[16] K. Tadaki, Algorithmic information theory and fractal sets. Proceedings of 1999 Workshop on Information-Based Induction Sciences (IBIS'99), pp. 105-110, August 26-27, 1999, Syuzenji, Shizuoka, Japan. In Japanese.

[17] K. Tadaki, "A generalization of Chaitin's halting probability $\Omega$ and halting self-similar sets," Hokkaido Math. J., vol. 31, pp. 219-253, 2002. Electronic Version Available: http://arxiv . org/abs/nlin/0212001v1

[18] K. Tadaki, A statistical mechanical interpretation of instantaneous codes. Proceedings of 2007 IEEE International Symposium on Information Theory (ISIT2007), pp. 1906-1910, June 24-29, 2007, Nice, France.

[19] K. Tadaki, A statistical mechanical interpretation of algorithmic information theory. Local Proceedings of Computability in Europe 2008 (CiE 2008), pp. 425-434, June 15-20, 2008, University of Athens, Greece. Extended and Electronic Version Available: http://arxiv . org/abs/0801.4194v1 
[20] K. Tadaki, The Tsallis entropy and the Shannon entropy of a universal probability. Proceedings of the 2008 IEEE International Symposium on Information Theory (ISIT 2008), pp. 2111-2115, July 6-11, 2008, Toronto, Canada.

[21] M. Toda, R. Kubo, and N. Saitô, Statistical Physics I. Equilibrium Statistical Mechanics, 2nd ed. Springer, Berlin, 1992.

[22] K. Weihrauch, Computable Analysis. Springer-Verlag, Berlin, 2000.

\section{A The proof of Theorem 3.4}

For completeness, we prove here Theorem 3.4, based on Theorems 4.6, 4.8, and 4.9, in a similar manner to the proof of Theorem 4.1 given in Section 5 .

Let $f:(0,1) \rightarrow \mathbb{R}$ with $f(T)=Z(T)$, and let $g:(0,1) \times \mathbb{N}^{+} \rightarrow \mathbb{R}$ with $g(T, k)=Z_{k}(T)$. First, it follows that $f$ is increasing.

Obviously, $\lim _{k \rightarrow \infty} g(T, k)=f(T)$ for every $T \in(0,1)$. It follows that, for every $T \in(0,1)$ and every $k \in \mathbb{N}^{+}$,

$$
g(T, k+1)-g(T, k)=2^{-\frac{\left|p_{k+1}\right|}{T}} .
$$

Thus, for every $T \in(0,1)$ and every $k \in \mathbb{N}^{+}, g(T, k)<g(T, k+1)$ and therefore $g(T, k)<f(T)$. At this point, the conditions (i), (ii), and (iv) of Theorem 4.6, all conditions of Theorem 4.8, and the conditions (i), (ii), (iv), (v), and (vi) of Theorem 4.9 hold for $g$.

Using the mean value theorem we see that, for every $k \in \mathbb{N}^{+}$and every $T, x, t \in(0,1)$ with $T<x<t$

$$
\frac{\ln 2}{t^{2}} W_{k}(T)(x-T)<g(x, k)-g(T, k)<\frac{\ln 2}{T^{2}} W_{k}(t)(x-T),
$$

where $W_{k}(T)=\sum_{i=1}^{k}\left|p_{i}\right| 2^{-\frac{\left|p_{i}\right|}{T}}$, as defined before Lemma 4.5 in Section 4 . Note that, for every $t \in(0,1), W_{k}(t)$ is increasing on $k$, and $W(t)=\lim _{k \rightarrow \infty} W_{k}(t)$ exists by Lemma 4.5 (i). Thus we see that

$$
\frac{\ln 2}{t^{2}} W_{1}(T)(x-T)<g(x, k)-g(T, k)<\frac{\ln 2}{T^{2}} W(t)(x-T),
$$

for every $k \in \mathbb{N}^{+}$and every $T, x, t \in(0,1)$ with $T<x<t$. Therefore, the condition (iii) of Theorem 4.6 and the condition (iii) of Theorem 4.9 hold for $g$.

Thus, Theorem 4.6, Theorem 4.8, and Theorem 4.9 result in the following three theorems, respectively.

Theorem A.1. For every $T \in(0,1)$, if $Z(T)$ is right-computable then $T$ is weakly Chaitin $T$ random.

Theorem A.2. For every $T \in(0,1)$, if $Z(T)$ is right-computable then $T$ is also right-computable.

Theorem A.3. For every $T \in(0,1)$, if $Z(T)$ is left-computable and $T$ is right-computable, then $T$ is T-compressible.

Theorem 3.4 follows immediately from these three theorems. 